\title{
Quaderni
}

QUADERNI Communication, technologies, pouvoir

86 | Hiver 2014-2015

Penser la politique par le film

\section{Avant-propos : penser le politique par le film}

\section{Laurent Godmer et David Smadja}

\section{(2) OpenEdition}

\section{Journals}

Édition électronique

URL : http://journals.openedition.org/quaderni/857

DOI : 10.4000/quaderni.857

ISSN : 2105-2956

\section{Éditeur}

Les éditions de la Maison des sciences de l'Homme

\section{Édition imprimée}

Date de publication : 5 janvier 2015

Pagination : 5-8

\section{Référence électronique}

Laurent Godmer et David Smadja, «Avant-propos : penser le politique par le film », Quaderni [En ligne], 86 | Hiver 2014-2015, mis en ligne le 05 janvier 2015, consulté le 23 septembre 2020. URL : http:// journals.openedition.org/quaderni/857 ; DOI : https://doi.org/10.4000/quaderni.857 


\section{$D$ ossier}

\section{Avant-propos : penser le politique \\ par le film}

En proposant de s'intéresser aux rapports entre politique et cinéma, ce numéro aborde un champ de recherche original peu défriché en science politique. Ce dossier se situe dans le prolongement des publications de dossiers consacrés à cette question notamment dans les revues Politix en $2003^{1}$ et Raisons politiques en $2010^{2}$. Au vu des travaux menés dans le cadre de la $58^{\mathrm{e}}$ Section thématique du XII ${ }^{\mathrm{e}}$ congrès de l'Association française de science politique (AFSP) qui a été organisée à Paris le 8 et le 10 juillet 2013, l'étude du politique au prisme de sa représentation cinématographique et filmique constitue un champ de recherche essentiellement

Laurent Godmer

\author{
Maître de conférences \\ en science politique \\ Université Paris-Est
}

David Smadja

Maître de conférences en science politique Université Paris-Est investi de manière individuelle et exploratoire. En effet, les outils théoriques et analytiques (Pierre Bourdieu, Stanley Cavell, Erving Goffman, etc.) et les matériaux utilisés (films particuliers, corpus de films, articles de presse, courriers des lecteurs, etc.) sont particulièrement divers. Il est pourtant bien établi que le cinéma, « au contraire », s'est fort tôt saisi de « la » politique. L'Affaire Dreyfus, réalisé par Georges Méliès, sorti en 1899 est ainsi parfois considéré comme le premier film de fiction de l'histoire du cinéma « politique ${ }^{3}$ ». La première version de ce film fut d'ailleurs « retirée des salles, en raison des empoignades qu'elle suscit $[\mathrm{a}]^{4}$ ». Or, ce tâtonnement analytique et méthodologique de la pensée du politique par l'étude des «films » lato sensu peut s'expliquer par le caractère contreintuitif de l'association entre « film » et « politique ». En effet, pourquoi s'intéresser aux films pour rendre compte de la signification du politique ? A priori, les films constituent des productions artistiques émanant d'un autre monde social qui n'entretient que peu de rapports avec celui des professionnels de la politique, des citoyens ou des 
groupes d'intérêt et qui obéit à d'autres logiques et contraintes que celles qui prévalent dans le champ politique (politics) ou dans le domaine de l'action publique (policy). Bien entendu leur contenu peut être politique au sens où ils peuvent « raconter des histoires » qui mettent en scène des personnages au sein d'institutions politiques ou bien des moments de campagne électorale, de conflit et de violence politique. Mais, dans ce cas, ils ne constituent en général que des contenants extérieurs au politique ${ }^{5}$ qui les instrumentalise en les exploitant comme vecteurs idéologiques, ou à l'inverse que les films peuvent instrumentaliser en le transformant en simple décor d'une action dont la signification est ailleurs ${ }^{6}$.

Pour autant, vu sous un autre angle, le rapport entre film et politique peut se présenter sous un jour beaucoup moins contingent. En effet, si l'on définit la politique comme ce qui est commun aux hommes dans leur pluralité et qui les relie les uns aux autres - en reprenant par exemple la définition de Hannah Arendt qui évoque un espace entre les hommes ${ }^{7}-$, il est possible de voir dans le cinéma l'une «des formes de la visibilité qui structurent un monde commun ${ }^{8} \gg$ et qui permettent de dévoiler avec les moyens qui leur sont propres un ordre composant en son sein les individus et les groupes et plus généralement « les personnes et des objets ${ }^{9} »$. Dit autrement, et avec les mots de Claude Lefort, ce n'est pas la politique qui importe, au sens de l'activité des professionnels de la politique, mais le politique qui " se révèle dans ce double mouvement d'apparition et d'occultation du mode d'institution de la société. ${ }^{10} »$. Par exemple, le cinéma correspond à une monstration indissociable d'une qualification technique du réel et donc d'un engagement d'objectivation. Les travaux d'André Bazin ont mis en évidence le réalisme ontologique (l'effet de réalité) inhérent à l'utilisation du dispositif cinématographique de la boîte noire et de la caméra qui engage une reproduction objective et impersonnelle d'une image « dont l'homme est exclu ». Pour cette raison, tout film, réaliste ou non, résulte d'une « mécanique impassible ${ }^{11}$ » qui contribue à fixer la croyance ${ }^{12}$ et qui irrigue un sens commun. En témoigne ce propos méthodologique de Madeleine Grawitz pour laquelle le document cinématographique affecte, par son réalisme même, la « nature » et la « portée » de cette information en permettant de reconstituer de manière plus fidèle le ton, la mimique, le geste et l'expression, autrement dit la vie elle-même ${ }^{13}$. En ce sens, le traitement filmique concourt à l'organisation, à la mise en scène et à la prescription du réel et par conséquent du souhaitable, contribuant ainsi à la construction d'un champ des possibles et d'un «partage du sensible ${ }^{14}$ ». En outre, il convient de reprendre un des célèbres aphorismes de Jean-Luc Godard - « Le cinéma est une pensée qui prend forme, une forme qui pense $^{15} \gg-$ qui invite à comprendre que le cinéma « aussi » pense spécifiquement et à sa manière le politique et partant que la politologie a un monde herméneutique à gagner en se nourrissant des travaux de la filmologie.

Ce dossier vise ainsi à appréhender le « film » de fiction (cinéma ou série) comme forme de visibilité du commun. Les recherches proposées dans ce dossier ont en commun d'appréhender la dimension politique des films. À travers les éléments de ce corpus, la narration filmique correspond à une restitution par l'image du commun et du lien qui existe entre les humains. 
Symétriquement, elle formule une critique par l'image des modes d'existence qui font obstacle au régime de l'entre-deux propre à la communauté spécifiquement politique et qui crée, de ce fait, une forme d'aliénation.

Quatre articles proposent ici autant de manières de voir comment les films pensent ce monde commun.

Les deux premiers articles abordent les films dans une articulation serrée avec les publics auxquels ils s'adressent. Boris Gobille, étudie Pierrot le fou, en l'abordant comme un « film question » permettant de reconstituer, au travers de controverses éthiques et politiques, des mises en visibilité du commun antagoniques variant autour de la question de savoir s'il est légitime « de traiter de la politique en esthète ». En s'intéressant à la représentation cinématographique des spectateurs des films qui en assurent la réception critique à travers le courrier des lecteurs, Boris Gobille se détourne de la figure omniprésente de l'artiste pour sociologiser le film en montrant de quelle manière le déchiffrement de l'œuvre correspond à une pratique de la vie ordinaire. Dans le même ordre d'idées, le choix des séries dans l'article de Antoine Faure et Emmanuel Taïeb permet une lecture sociologique des fictions qui coïncident avec la vie quotidienne du public par leur caractère itératif et régulier. Il n'est d'ailleurs pas indifférent de rappeler que l'intérêt des sciences sociales pour les fictions a débuté avec l'émergence des séries télévisées. Prenant acte du développement de séries télévisées américaines possédant un niveau d'écriture de « grande qualité », les auteurs montrent comment cette forme narrative contribue à discuter l'ordre politique des sociétés occidentales, voire à le subvertir. En particulier, par la représentation du temps social, les séries fournissent une refondation continue de la communauté et, ce faisant, elles revêtent une signification politique au sens où l'entend Jacques Rancière.

Les deux articles suivants s'inscrivent dans un axe privilégiant la pensée politique des réalisateurs et une approche davantage interne des productions, davantage orientée vers la signification des films que vers le type de communication qu'ils instituent avec leur public. Tout d'abord, Gwendal Châton s'intéresse au cinéma d'un autre réalisateur consacré, Clint Eastwood, et montre de quelle manière il constitue un vecteur discret, mais redoutablement efficace, d'une philosophie politique marginale et radicale, la pensée libertarienne. Renvoyant dos-à-dos engagement idéologique néo-conservateur et désintéressement artiste, Gwendal Châton met au jour l'activité d'un cinéaste qui pense le politique $^{16}$ à partir de l'individu et rend visible le commun à partir de la pluralité en articulant « utopies communautaires » et « individualisme altruiste ». Enfin, Charles Bosvieux analyse le film de Pierre Schœller, L'Exercice de l'État, en s'intéressant au parti pris de filmage qui consiste à saisir la logique étatique au travers des objets en apparence les plus insignifiants (une circulaire, une commission, un parapheur) en conformité avec l'approche sociologique de Pierre Bourdieu dans Sur l'État. En montrant en particulier la centralité de la communication politique, Pierre Schœller dévoile une pratique mutilée du commun. Il formule une critique de la « démocratie des technocrates » qui éprouve, plus qu'elle ne ratifie, la définition arendtienne du politique comme monde commun. 
$\mathrm{N} \cdot \mathrm{O} \cdot \mathrm{T} \cdot \mathrm{E} \cdot \mathrm{S}$

1. Politix, dossier « Politiques du cinéma », vol. 16, $\mathrm{n}^{\circ} 61,1^{\text {er }}$ trimestre 2003. Voir également dans le domaine de la sociologie, Jean-Pierre Esquenazi, « Le film, un fait social », Réseaux, 2000/1, n 99.

2. Raisons politiques, dossiers « Cinématographies du politique », vol. 1 et 2, numéros 38 et 39, 2010 (dir. David Smadja).

3. Fabien Danesi, «Politique», in Antoine de Baecque et Philippe Chevallier (dir.), Dictionnaire de la pensée du cinéma, Paris, Presses universitaires de France, 2012, pp. 548-550.

4. Ibid., p. 548.

5. Voir Laurent Garreau, Les archives secrètes $d u$ cinéma français, Paris Presses universitaires de France, 2009 ; Sylvie Lindeperg, « La Résistance rejouée, Usages gaullistes du cinéma », Politix, 1993, vol. 6, $\mathrm{n}^{\circ} 24$, pp. 134-152.

6. Jean-Pierre Esquenazi, « Le film, un fait social », Réseaux, 2000, vol. 18, n 99, p. 15.

7. Hannah Arendt, Qu'est-ce que la politique?, Paris, Le Seuil, 2001, trad. fr. Sylvie Courtine-Denamy, indique : «La politique traite de la communauté et de la réciprocité d'être différents » (p. 40), «[elle] prend naissance dans l'espace-qui-est-entre- les hommes [...]. » (ibid.., p. 42). .

8. Jacques Rancière, «Le cinéma, un art contrarié », Et tant pis pour les gens fatigués, Entretiens, Paris, Éditions Amsterdam, 2009.

9. Robert Bresson, Notes sur le cinématographe, Paris, Gallimard, 1995, p. 28.

10. Claude Lefort, Essai sur le politique. XIX $X^{e}-X X^{e}$ siècle, Paris, Le Seuil, 2001. Sur ce point voir également M. Coulomb-Guly, Jean-Pierre Esquenazi, «Fiction et politique : doubles jeux », Mots, Les langages du politique, 2012/2., $\mathrm{n}^{\circ} 99$.
11. André Bazin, Qu'est-ce que le cinéma?, Paris, Le Cerf, 1975.

12. Charles S. Peirce, « Comment se fixe la croyance?», in Textes anticartésiens, Paris, Aubier, 1984, p. 275.

13. Madeleine Grawitz, Méthodes des sciences sociales, Paris, Dalloz, 2001, pp. 394-395 et pp. 598600.

14. Jacques Rancière, Le partage du sensible : Esthétique et politique, Paris, La Fabrique, 2000.

15. Cité par Antoine de Baecque et Philippe Chevallier, « Avant-propos », in Antoine de Baecque et Philippe Chevallier (dir.), op. cit., p. VII. Sur Jean-Luc Godard, voir Antoine de Baecque, Godard, Paris, Grasset, 2012. 16. Pour un autre exemple, voir Laurent Godmer, «Science politique et cinéma : penser le politique et le local avec Éric Rohmer », Raisons politiques, n³8, mai 2010, pp. 17-30. Sur Rohmer, voir Antoine de Baecque et Noël Herpe, Éric Rohmer, biographie, Paris, Stock, 2014. 